\title{
Current Understanding of the Biology of Vascular Endothelium
}

\author{
Yasufumi Sato \\ Department of Vascular Biology, Institute of Development, Aging and Cancer, Tohoku University, 4-1 Seiryo- \\ machi, Aoba-ku, Sendai 980-8575, Japan
}

The vascular wall is composed of endothelial cells and mural smooth muscle cells/pericytes. The vascular endothelium is a continuous monolayer of endothelial cells lining the inner surface of blood vessels. Vascular endothelium is not merely a blood-compatible lining for blood cells and plasma, but is metabolically active and plays principal roles in regulating vascular permeability, macromolecular transport, vascular tone, inflammation, coagulation, and vascular wall structure.

Endothelial cell-cell contacts control the permeability of the blood vessel wall. This allows the vascular endothelium to form a barrier for solutes, macromolecules, and blood cells between the vessel lumen and the interstitial space. Communication between blood and tissue occurs through the delivery of macromolecules and circulating substances across the endothelial barrier by directed transport either through or between endothelial cells.

The mechanical environment has an influence on the structure and function of various cell types. For the vascular endothelium located at the interface of the flowing blood and the underlying vessel wall, the importance of blood flow and associated wall shear stress is obvious. Not only is it a sensitive regulator of endothelial structure and function, but it will also be influenced by different flow environments.

It is also evident that the vascular endothelium contributes to the local control of vascular tone by releasing potent vasodilators and vasoconstrictors. Under physiological conditions, the vascular endothelium continuously releases nitric oxide (NO), which relaxes surrounding smooth muscle cells and ensures vessel patency. Activated vascular endothelium can also secrete vasoconstrictors, the best known of which is endothelin-1 (ET-1), as well as other factors that affect the differentiation and growth of vascular smooth muscle cells.

The vascular endothelium is pivotal in the control of hemostasis and thrombosis because it is the primary source of the major hemostatic regulatory molecules. Normally,

Department of Vascular Biology, Institute of Development, Aging and Cancer, Tohoku University, 4-1 Seiryo-machi, Aoba-ku, Sendai 980-8575, Japan.

Tel: +81-22-717-8528, Fax: +81-22-717-8533

E-mail: y-sato@idac.tohoku.ac.jp endothelial cells are anticoagulant and antithrombotic. This is due to the secretion of antiplatelet substances, including $\mathrm{PGI}_{2}$ and NO. Following vessel injury, local generation of thrombin at the site of endothelial denudation causes a series of coagulation responses, while the surface of the intact vascular endothelium is the site for inactivation of thrombin by antithrombin, and its conversion to a coagulation inhibitor by interaction with thrombomodulin. The vasular endothelium is also the source of tissue-type plasminogen activator for thrombolysis.

A particular role in inflammation is played by cell adhesion molecules (CAMs), expressed both on the endothelial and blood cells. In inflammation, white blood cells interact with the vascular endothelium by a spatio-temporal intervention pattern of specific cell surface receptors and adhesion molecules. The resident vascular endothelium of the inflammatory region communicates with circulating white blood cells by inflammatory cytokines and direct cell-cell contact. These cytokines stimulate expression of adhesion molecules such as ICAM-1, VCAM-1, and E- and P-selectin on the endothelial surface and up-regulate integrins and ICAM-1 on white blood cells. White blood cells then adhere to the activated endothelium, migrate through the vessel wall, and penetrate areas of infection or tissue damage.

Vascular endothelial cells are normally quiescent, but have the ability to actively proliferate and form neovessels. Angiogenesis is a process by which new blood vessels are formed from pre-existing ones, and is a fundamental process in reproduction and development. The vascular system is the first functional organ that develops in the vertebrate embryo. Blood vessels of the entire body are formed via two distinctive processes, vasculogenesis and angiogenesis. The initial process of vascular development is vasculogenesis. In this process, hemangioblasts or angioblasts of mesoderm origin differentiate into endothelial cells and form a primitive vascular plexus. In a subsequent process of angiogenesis, neovessels form from extant primitive vessels and distribute to the entire body. In adults, angiogenesis is observed only in restricted places such as the endometrium and ovarian follicle, and this process is normally transient.

The functions of vascular endothelium described above are sensitive to various stimuli including cytokines, oxidized-LDL, hypoxia, endotoxin, or mechanical stress. In 
response to these stimuli, endothelial cells become activated, disrupt barrier function, and enhance vasoconstriction, coagulation, leukocyte adhesion, and proliferation. Although these responses likely exist as protective mechanisms, once the stimuli are severe the responses may become excessive, resulting in damaged vessels, impaired organ function, and abnormal proliferative response.
This review cluster will focus on the current understanding of endothelial cell biology in terms of the molecules and biochemical pathways involved. These regulate vascular tone, hence blood flow and pressure; changes in permeability and leukocyte traffic during the generation of inflammatory and immune responses; and finally the processes of vessel growth and angiogenesis. 